\title{
Capacity-Delay Tradeoff for Information Dissemination Modalities in Wireless Networks
}

\author{
Zheng Wang $^{\dagger}$, Hamid R. Sadjadpour ${ }^{\dagger}$, J.J. Garcia-Luna-Aceves ${ }^{\ddagger}$ \\ Department of Electrical Engineering ${ }^{\dagger}$ and Computer Engineering ${ }^{\ddagger}$ \\ University of California, Santa Cruz, 1156 High Street, Santa Cruz, CA 95064, USA \\ $\ddagger$ Palo Alto Research Center (PARC), 3333 Coyote Hill Road, Palo Alto, CA 94304, USA \\ Email:\{wzgold, hamid, jj\}@soe.ucsc.edu
}

\begin{abstract}
This paper presents the first comprehensive capacity-delay tradeoff study for random wireless ad hoc networks under all information dissemination modalities (unicast, multicast, broadcast, anycast) when nodes operate either with multi-packet reception (MPR) or single-packet reception (SPR) capabilities.

Our results demonstrate that for unicast, increasing capacity requires additional delay for SPR similar to the results in [1] while MPR incurs no penalty, i.e., we can increase capacity and decrease delay simultaneously for MPR. For multicast, there is no tradeoff for both SPR and MPR. However, similar tradeoff can be observed for broadcast when MPR is used while there is no tradeoff with SPR.
\end{abstract}

\section{INTRODUCTION}

The seminal work by Gupta and Kumar [2] on the scaling laws of wireless ad hoc networks for unicast with multihop communication demonstrate that when nodes only receive a single packet (SPR), the capacity decreases as the number of nodes in the network increases. As a result, many research activities focused on improving this capacity for unicast [3], [4]. Furthermore, there are many contributions in computing the scaling laws in wireless ad hoc networks for multicast [5] and broadcast [6]. Our first contribution focuses in developing a unifying approach to compute different information dissemination modalities (unicast, multicast, broadcast, anycast) for multi-packet reception (MPR) technique based on the results of point-to-point single-packet reception (SPR) technique derived in [7].

Another important area of research is related to the capacitydelay tradeoff study [1] in wireless ad hoc networks. However, there is no contribution in literature to study this tradeoff for all kinds of communications. Our next contribution in this paper relates to introducing the first tradeoff study between capacity and delay for different information dissemination modalities and when nodes are endowed with SPR or MPR capabilities.

Multi-packet reception (MPR) is a cooperative technique [8] that allows multiple nodes to transmit their packets simultaneously to the same receiver node, which can in turn decode all such packets successfully. There are many ways for transmission cooperation such as multiuser detection (MUD), directional antennas, or multiple input multiple output (MIMO) techniques. MPR has been shown to increase the capacity regions of ad hoc networks, and recently Garcia-Luna-Aceves et al. [4] have shown that the order capacity of ad hoc networks subject to multi-pair unicast traffic is increased with MPR. However, no capacity results have been reported on the benefits of MPR in networks subject to other classes of information dissemination.

The paper is organized as follows. Section II describes the assumptions and definitions that we use throughout the paper. In Section III, we compute a tight bound for the capacity of wireless ad hoc networks with MPR. Section IV describes the delay computation together with capacity-delay tradeoff for all information dissemination modalities when nodes are endowed with MPR or SPR. In Section V, we discuss the results derived earlier and their implications.

\section{Network Model And Preliminaries}

Our capacity analysis is based on the protocol model for dense networks introduced by Gupta and Kumar [2]. The case of what we call SPR corresponds to the original protocol model, and we make an extension to account for MPR capability at the receivers.

Definition 2.1: The Protocol Model for SPR: All nodes use a common transmission range $r(n)$ for all their communications. The network area is assumed to be a unit square area. Node $X_{i}$ can successfully transmit to node $X_{j}$ if for any node $X_{k}, k \neq i$, that transmits at the same time as $X_{i}$, then $\left|X_{i}-X_{j}\right| \leq r(n)$ and $\left|X_{k}-X_{j}\right| \geq(1+\Delta) r(n)$.

In wireless networks with MPR capability, the protocol model assumption allows simultaneous decoding of packets for all nodes as long as they are within a radius of $R(n)$ from the receiver and all other transmitting nodes have a distance larger than $(1+\Delta) R(n)$. The difference is that we allow the receiver node to receive multiple packets from different nodes within its disk of radius $R(n)$ simultaneously [4]. Note that $r(n)$ in Gupta and Kumar's model is a random variable while $R(n)$ in MPR is a predefined value which depends on the complexity of receivers. We assume that nodes cannot transmit and receive at the same time, which is equivalent to half duplex communications [2]. The data rate for each transmitter-receiver pair is a constant value of $W$ bits/second and does not depend on $n$. Given that $W$ does not change the order capacity of the network, we normalize its value to one. The relationship between receiver range of MPR throughout this paper and transmission range in [2] is 
defined as $R(n)=r(n) \geq \Theta(\sqrt{(\log n) / n})$ to guarantee the connectivity criterion [2].

Definition 2.2: $(n, m, k)$-cast tree: An $(n, m, k)$-cast tree is a set of nodes that connects a source node of an $(n, m, k)$ cast with all its intended $k$ receivers out of $m$ choices $(k \leq m \leq n)$, in order for the source to send information to $k$ of those receivers. By this definition, it can be seen that $(n, 1,1),(n, m, m),(n, n, n)$, and $(n, m, k)$ are unicast, multicast, broadcast, and anycast respectively.

Definition 2.3: Feasible throughput capacity of $(n, m, k)$ cast: In a wireless ad hoc network of $n$ nodes in which each source node transmits its packets to $k$ out of $m$ destinations, a throughput of $\lambda_{m, k}(n)$ bits per second for each node is feasible if there is a spatial and temporal scheme for scheduling transmissions, such that by operating the network in a multihop fashion and buffering at intermediate nodes when awaiting transmission, every node can send $\lambda_{m, k}(n)$ bits per second on average to its $k$ out of its $m$ chosen destination nodes. That is, there is a $T<\infty$ such that in every time interval $[(i-1) T, i T]$ every node can send $T \lambda_{m, k}(n)$ bits to its corresponding destination nodes.

Definition 2.4: Order of throughput capacity: $C_{m, k}(n)$ is said to be of order $\Theta(f(n))$ bits per second if there exist deterministic positive constants $c$ and $c^{\prime}$ such that

$$
\left\{\begin{array}{l}
\lim _{n \rightarrow \infty} \operatorname{Prob}\left(C_{m, k}(n)=c f(n) \text { is feasible }\right)=1 \\
\lim _{n \rightarrow \infty} \operatorname{Prob}\left(C_{m, k}(n)=c^{\prime} f(n) \text { is feasible }\right)<1 .
\end{array}\right.
$$

Keshavarz et al. [6] used Maximum Independent Set $\operatorname{MIS}(\Delta, r(n))$ to describe the maximum number of simultaneous transmitters and Minimum Connected Dominating Set $\operatorname{MCDS}(r(n))$ for computing the minimum rebroadcasting times required to reach the destinations in a network when nodes use SPR and broadcasting. For the same purpose and to account for the use of MPR and $(n, m, k)$-cast, we define the Maximum MPR Independent Set $(\operatorname{MMIS}(\Delta, R(n)))$ and the Minimum Euclidean $(n, m, k)$-cast Tree $(\operatorname{MEMKT}(R(n)))$ definitions.

Definition 2.5: Maximum MPR Independent Set $(\operatorname{MMIS}(\Delta, R(n)))$ : An MPR independent set is a set of nodes in a graph $G$ that contains one receiver node and all (transmitting) nodes within a distance of $R(n)$ from this receiver node. A Maximum MPR Independent Set $(\operatorname{MMIS}(\Delta, R(n)))$ consists of the maximum number of MPR sets that simultaneously transmit their packets while MPR protocol model is satisfied for all these MPR sets. If we add any transmitter node from $G$ to $\operatorname{MMIS}(\Delta, R(n))$, there is at least one MPR set that violates the MPR protocol model.

Definition 2.6: Minimum Euclidean ( $n, m, k)$-cast Tree $(\operatorname{MEMKT}(R(n)))$ : The $\operatorname{MEMKT}(R(n))$ is an $(n, m, k)$-cast tree in which the $k$ destinations out of $m$ nodes receive information from the source and this $(n, m, k)$-cast tree has the minimum total Euclidean distance. For example, when $k=m$, $\operatorname{MEMKT}(R(n))$ denotes the minimum Euclidean multicast tree, $(\operatorname{MEMT}(R(n)))$, that is defined in graph theory.
Following the assumption in [1], [2], we assume that the packet size is small enough so that the packet delay is essentially equal to the number of hops taken by the packet.

Definition 2.7: Delay in $(n, m, k)$-cast communication In $(n, m, k)$-cast, the delay of a packet in a network is the time it takes the packet to reach every destination after it leaves the source. We do not take queuing delay at the source into account, since our interest is in the network delay. The average packet delay for a network with $n$ nodes $D_{m, k}(n)$, is obtained by averaging over all packets, all source-destination pairs, and all random network configurations.

For the rest of this paper, $\|T\|$ denotes the total Euclidean distance of a tree $T$; $\# T$ is used to denote the total number of vertices (nodes) in a tree $T$; and $\overline{\|T\|}$ is used for the statistical average of the total Euclidean distance of a tree.

\section{The CAPACITY OF $(n, m, k)$-CAST WITH MPR}

\section{A. Upper Bound}

The following Lemma provides an upper bound in terms of the ratio of the size of $\operatorname{MMIS}(\Delta, R(n))$ to the size of $\operatorname{MEMKT}(R(n))$. Essentially, $\overline{\# \operatorname{MEMKT}(R(n))}$ equals the minimum number of transmissions required to $(n, m, k)$-cast a packet to $k$ destinations out of $m$, and $\overline{\# \operatorname{MMIS}(\Delta, R(n))}$ represents the maximum number of successful simultaneous transmissions when MPR is used. The proof is similar to [7] and because of page limitation, it is not provided here.

Lemma 3.1: The per-node throughput capacity of $(n, m, k)$ cast with MPR is given by $O\left(\frac{1}{n} \times \frac{\overline{\# M M I S}(\Delta, R(n))}{\# \text { MEMKT }(R(n))}\right)$.

We next compute the upper bound of $\# \operatorname{MMIS}(\Delta, R(n))$ and the lower bound of \#MEMKT $(R(n))$. To compute the lower bound for $\# \operatorname{MEMKT}(R(n))$, we find the relationship between \#MEMKT $(R(n))$ and the total length of Euclidean Minimum Spanning Tree (EMST), $\overline{\|E M S T\|}$. Because of space limitation, the proof of this Lemma is omitted and similar proof can be found in [7].

Lemma 3.2: In $(n, m, k)$-cast applications, the average number of nodes in $\operatorname{MEMKT}(R(n))$ has the following lower bound as

$\overline{\text { \#MEMKT }(R(n))} \geq\left\{\begin{array}{l}\Theta\left(k(\sqrt{m} R(n))^{-1}\right) \text { for } m \leq \Theta\left(m_{b}\right) \\ \Theta(k) \quad \text { for } k \leq \Theta\left(m_{b}\right)<m \\ \Theta\left(R^{-2}(n)\right) \text { for } \Theta\left(m_{b}\right)<k \leq m\end{array}\right.$

where $m_{b}=R^{-2}(n)$.

Combining Lemmas 3.1, 3.2, and the obvious observation that $\overline{\# M M I S}(\Delta, R(n)) \leq \Theta(n)$, we can compute the upper bound capacity of $(n, m, k)$-cast with MPR in the following theorem.

Theorem 3.3: In dense random wireless ad hoc networks with MPR, the upper bound per-node throughput capacity of $(n, m, k)$-cast is

$$
C_{m, k}(n)=\left\{\begin{array}{l}
O\left(k^{-1} \sqrt{m} R(n)\right) \text { for } m \leq \Theta\left(m_{b}\right) \\
O\left(k^{-1}\right) \quad \text { for } k \leq \Theta\left(m_{b}\right)<m, \\
O\left(R^{2}(n)\right) \text { for } \Theta\left(m_{b}\right)<k \leq m
\end{array}\right.
$$

where $m_{b}=R^{-2}(n)$. 


\section{B. Lower Bound}

To derive an achievable lower bound, we use a TDMA scheme for random dense wireless ad hoc networks similar to the approach used in [9]. We first divide the network area into square cells which has been shown in Fig. 1. Under this condition, connectivity inside all cells is guaranteed and all nodes inside a cell are within communication range of each other. We build a cell graph over the cells that are occupied with at least one vertex (node). Two cells are connected if there exists a pair of nodes, one in each cell, that are less than or equal to $R(n)$ distance apart. Because the whole network is connected when $R(n)=r(n) \geq \Theta(\sqrt{\log n / n})$, it follows that the cell graph is connected [9]. Let $L$ represent the minimum number of cell separations in each group of cells that communicate simultaneously. The capacity reduction caused by the TDMA scheme is a constant factor and does not change the order capacity of the network.

Next we prove that, when $n$ nodes are distributed uniformly over a unit square area, we have simultaneously at least $\frac{1}{(L R(n) / \sqrt{2})^{2}}$ circular regions (see Fig. 1), each one containing $\Theta\left(n R^{2}(n)\right)$ nodes w.h.p..

Lemma 3.4: The circular area of radius $R(n)$ corresponding to the receiver range of a receiver $j$ contains $\Theta\left(n R^{2}(n)\right)$ nodes w.h.p., and is uniformly distributed for all values of $j, 1 \leq$ $j \leq \frac{1}{(L R(n) / \sqrt{2})^{2}}$.

Proof: From the Chernoff bound we used in [7], for any given $0<\delta<1$, we can find $\theta>0$ such that $P\left[\left|N_{j}-E\left(N_{j}\right)\right|>\delta E\left(N_{j}\right)\right]<e^{-\theta E\left(N_{j}\right)}$. Thus, we can conclude that the probability that the value of the random variable $N_{j}$ deviates by an arbitrarily small constant value from the mean tends to zero as $n \rightarrow \infty$. This is a key step in showing that when all the events $\bigcap_{j=1}^{\frac{1}{(L R(n) / \sqrt{2})^{2}}}\left|N_{j}-E\left(N_{j}\right)\right|<\delta E\left(N_{j}\right)$ occur simultaneously, then all $N_{j}$ 's converge uniformly to their expected values. Utilizing the union bound and $E\left(N_{j}\right)=$ $\pi n R^{2}(n)$, we arrive at

$$
\begin{aligned}
& \lim _{n \rightarrow \infty} P\left[\bigcap_{j=1}^{\frac{1}{(L R(n) / \sqrt{2})^{2}}}\left|N_{j}-E\left(N_{j}\right)\right|<\delta E\left(N_{j}\right)\right] \\
& \geq 1-\lim _{n \rightarrow \infty} \frac{1}{(L R(n) / \sqrt{2})^{2}} e^{-\theta \pi n R^{2}(n)}
\end{aligned}
$$

Utilizing the connectivity criterion, $\lim _{n \rightarrow \infty} \frac{e^{-\theta \pi n R^{2}(n)}}{R^{2}(n)} \rightarrow$ 0 , which finishes the proof.

Define \#MEMKTC $(R(n))$ as the total number of cells that contain all the nodes in an $(n, m, k)$-cast group. Next Lemma provides a tight bound for \#MEMKTC $(R(n))$.

Lemma 3.5: The average number of cells covered by the nodes in MEMKTC $(R(n))$, is tight bounded as follows:

$$
\overline{\text { \#MEMKTC }(R(n))}=\left\{\begin{array}{l}
\Theta\left(k(\sqrt{m} R(n))^{-1}\right) \text { for } m \leq \Theta(m) \\
\Theta(k) \quad \text { for } k \leq \Theta\left(m_{b}\right)<m \\
\Theta\left(R^{-2}(n)\right) \text { for } \Theta\left(m_{b}\right)<k \leq m
\end{array}\right.
$$

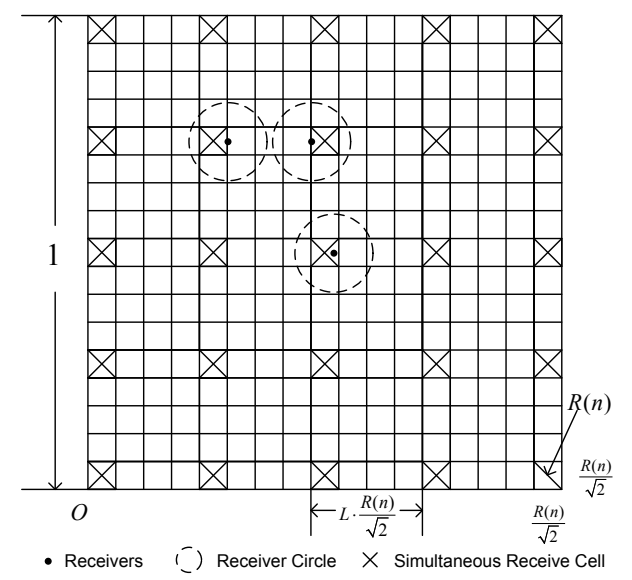

Fig. 1. Cell construction used to derive a lower bound on capacity

where $m_{b}=R^{-2}(n)$.

Proof: The proof for the tight bound of \#MEMKTC $(R(n))$ is similar to the proof of tight bound for \#MEMTC $(R(n))$ which can be found with details in [7].

Theorem 3.6: The achievable per node lower bound of the $(n, m, k)$-cast throughput capacity with MPR is

$$
C_{m, k}(n)=\left\{\begin{array}{l}
\Omega\left(k^{-1} \sqrt{m} R(n)\right) \text { for } m \leq \Theta\left(m_{b}\right) \\
\Omega\left(k^{-1}\right) \quad \text { for } k \leq \Theta\left(m_{b}\right)<m, \\
\Omega\left(R^{2}(n)\right) \text { for } \Theta\left(m_{b}\right)<k \leq m
\end{array}\right.
$$

where $m_{b}=R^{-2}(n)$.

Proof: There are $(R(n) / \sqrt{2})^{-2}$ cells in the unit square network area and only $(L R(n) / \sqrt{2})^{-2}$ of these cells can communicate simultaneously because of the TDMA scheme that we described earlier. From the definition of \#MEMKTC $(R(n))$, it is clear that there are in the order of \#MEMKTC $(R(n))$ transmissions required in order to transfer a packet from source to all its destinations in any $(n, m, k)$-cast communication scheme. It is clear from Lemma 3.4 that for each of $(L R(n) / \sqrt{2})^{-2}$ simultaneous transmitting cells, there are $\Theta\left(\pi R^{2}(n) n\right)$ nodes transmitting packets to their respected receiver nodes using MPR. Since each one of $(n, m, k)$-cast group requires \#MEMKTC $(R(n))$ transmissions, the total throughput capacity lower bound for the network is equal to $\Omega\left(\frac{(R(n) / \sqrt{2})^{-2} \times\left(\pi R^{2}(n) n\right)}{\overline{\text { MEMKKTC }(R(n))}}\right)$. If we divide this value by the total number of nodes in the network, $n$, and substitute \#MEMKTC $(R(n))$ with the results from Lemma 3.5, then the theorem will be proved.

\section{Capacity with MPR and SPR}

From Theorems 3.3 and 3.6, we can provide the tight bound throughput capacity for the $(n, m, k)$-cast when the node have MPR capability in dense random wireless ad hoc networks as follows.

Theorem 3.7: The throughput capacity of $(n, m, k)$-cast in 
a random dense wireless ad hoc network with MPR is

$$
C_{m, k}(n)= \begin{cases}\Theta\left(k^{-1} \sqrt{m} R(n)\right) \text { for } m \leq \Theta\left(m_{b}\right) \\ \Theta\left(k^{-1}\right) & \text { for } k \leq \Theta\left(m_{b}\right)<m, \\ \Theta\left(R^{2}(n)\right) & \text { for } \Theta\left(m_{b}\right)<k \leq m\end{cases}
$$

where $m_{b}=R^{-2}(n)$.

The throughput capacity for networks using SPR is given in [5] for the case of multicasting (i.e., $(n, m, m)$-cast). However, the results we just derived for the capacity of $(n, m, k)$-cast with MPR can be extended to address SPR as stated in the following theorem. Due to space limitations, we only present summary of the proof and the details can be found in [7].

Theorem 3.8: The throughput capacity of $(n, m, k)$-cast in a random dense wireless ad hoc network with SPR is

$$
C_{m, k}(n)=\left\{\begin{array}{l}
\Theta\left(\sqrt{m}(n k r(n))^{-1}\right) \text { for } m \leq \Theta\left(m_{b}\right) \\
\Theta\left(\left(n k r^{2}(n)\right)^{-1}\right) \quad \text { for } k \leq \Theta\left(m_{b}\right)<m, \\
\Theta\left(n^{-1}\right) \quad \text { for } \Theta\left(m_{b}\right)<k \leq m
\end{array}\right.
$$

where $m_{b}=r^{-2}(n)$.

Summary of proof: The proof follows the same approach used for the case of MPR, with two key differences. First, the receiver range $R(n)$ must be replaced with the transmission range $r(n)$ for SPR. Second, there can be at most a single successful transmission inside a circle of radius $r(n)$ centered around each receiver node.

Note that for the computation of capacity, we have computed three different regions of capacity both for the MPR and SPR approaches. These three regions of capacity are called unicast, multicast, and broadcast regions depending on the values of $m$ and $k$. In this paper, we did not show how to derive these three different regions due to space limitations. The interested reader can find the details for the SPR case in [7] and the proof for the MPR is similar.

\section{DElay WITH MPR AND SPR}

In this section, we present the result regarding the tradeoff between delay and capacity. As we defined earlier in definition 2.7, packet delay is proportional to the total number of hops required from each source to its destinations. In order to compute this delay, we first prove the following lemma.

Lemma 4.1: The delay of $(n, m, k)$-cast in a random dense wireless ad hoc network with MPR is

$$
D_{m, k}(n)=\Theta(\overline{\# \operatorname{MEMKTC}(R(n))})
$$

Proof: From the definition of $\overline{\text { \#MEMKTC }(R(n))}$ and Lemma 3.5, we conclude that \#MEMKTC $(R(n))$ is proportional to the minimum number of hops in which the information is routed from source to all its destinations. Since we are using a TDMA scheme to achieve the lower bound for the capacity, it is clear that in order to transport the information from one cell to the next adjacent cell, we need between one to two hops (see Fig. 1). Therefore, \#MEMKTC $(R(n))$ is also in the same order as the total number of hops. Based on the definition of delay, it is clear that \#MEMKTC $(R(n))$ is also the same order bound as the total delay which proves the Lemma.

Lemma 4.1 can be similarly extended to SPR to compute the delay and due to page limitations, we simply omit the results.

El Gamal et. al [1] demonstrated the tradeoff relationship between capacity and delay only for SPR and the multi-pair unicast case. Here, we extend this relationship for the general $(n, m, k)$-cast and both for SPR and MPR cases. Our result corroborates the unicast with SPR [1] as well as provides new results for all other cases of communications in wireless ad hoc networks.

Theorem 4.2: The relationship between capacity and delay for $(n, m, k)$-cast with MPR is given below and shown in Table I.

$$
C_{m, k}(n) D_{m, k}(n)=\Theta(1)
$$

Proof: The results can be easily derived by comparing Theorem 3.7 with Lemmas 4.1 and 3.5.

TABLE I

THE RELATIONSHIP BETWEEN CAPACITY AND DELAY WITH MPR

\begin{tabular}{|c|c|c|}
\hline & $D_{m, k}(n)$ & $C_{m, k}(n)$ \\
\hline$k \leq m \leq \Theta\left(R^{-2}(n)\right)$ & $\Theta\left(\frac{k}{\sqrt{m} R(n)}\right)$ & $\Theta\left(\frac{\sqrt{m} R(n)}{k}\right)$ \\
\hline$k \leq \Theta\left(R^{-2}(n)\right) \leq m$ & $\Theta(k)$ & $\Theta\left(\frac{1}{k}\right)$ \\
\hline$\Theta\left(R^{-2}(n)\right) \leq k \leq m$ & $\Theta\left(R^{-2}(n)\right)$ & $\Theta\left(R^{2}(n)\right)$ \\
\hline
\end{tabular}

Theorem 4.3: The relationship between capacity and delay in $(n, m, k)$-cast with SPR is given below and shown in Table II.

$$
C_{m, k}(n) D_{m, k}(n)=\left(n r^{2}(n)\right)^{-1}
$$

TABLE II

THE RELATIONSHIP BETWEEN CAPACITY AND DELAY WITH SPR

\begin{tabular}{|c|c|c|}
\hline & $D_{m, k}(n)$ & $C_{m, k}(n)$ \\
\hline$k \leq m \leq \Theta\left(r^{-2}(n)\right)$ & $\Theta\left(\frac{k}{\sqrt{m} r(n)}\right)$ & $\Theta\left(\frac{\sqrt{m}}{n k r(n)}\right)$ \\
\hline$k \leq \Theta\left(r^{-2}(n)\right) \leq m$ & $\Theta(k)$ & $\Theta\left(\frac{1}{n k r^{2}(n)}\right)$ \\
\hline$\Theta\left(r^{-2}(n)\right) \leq k \leq m$ & $\Theta\left(r^{-2}(n)\right)$ & $\Theta\left(n^{-1}\right)$ \\
\hline
\end{tabular}

The capacity-delay tradeoff in [1] is a special case of our results for $m=k=1$ which can be shown as $D_{1,1}(n)=$ $\Theta\left(n C_{1,1}(n)\right)$.

\section{Discussion of Results And Conclusions}

Theorems 3.8 and 3.7 provides capacity information for SPR and MPR respectively. There are three different capacity regions depending on the values of $k$ and $m$ in $(n, m, k)$ cast. Fig. 2 compares the tradeoff between throughput capacity and delay for MPR and SPR for all these three regions of capacity. By observing the capacity for MPR and SPR, we notice that the receiver range $R(n)$ is multiplied for capacity computation in MPR in two regions in Eq. (7) and in one region is independent of $R(n)$ while the transmission range $r(n)$ is divided for capacity computation in SPR in the first two regions of capacity. This behavior is demonstrated in Fig. 


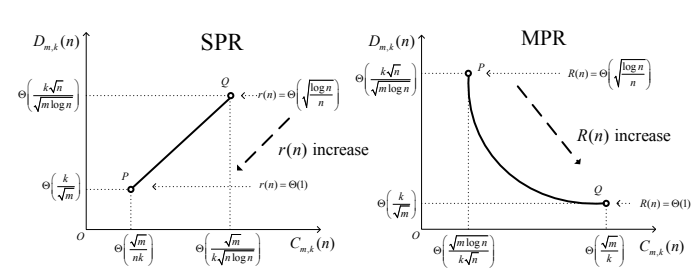

(a) $k \leq m \leq \Theta\left(\frac{1}{r^{2}(n)}\right)$

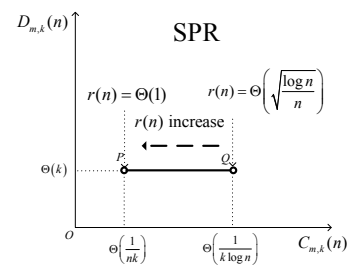

(b) $k \leq \Theta\left(\frac{1}{r^{2}(n)}\right) \leq m$
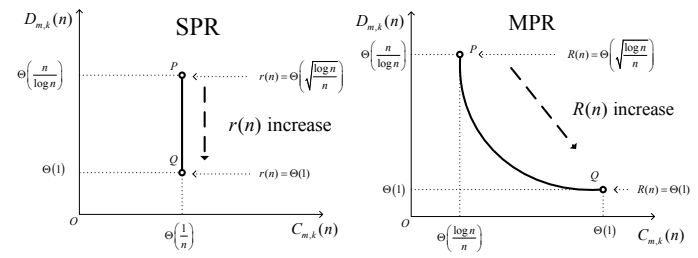

(c) $\Theta\left(\frac{1}{r^{2}(n)}\right) \leq k \leq m$

Fig. 2. The tradeoff between capacity and delay

2. This fundamental difference is due to the fact that the MPR scheme embraces interference, while SPR is based on avoiding interference by limiting transmission range.

The above result indicates that large capacity increases can be attained by embracing interference with MPR and embracing opportunism by appropriate use of in-network storage and information dissemination from the nearest site(s) of a communication group, rather than from pre-defined origins hosting the content. If the communication group is the entire network $(m=n)$, information flows from the closest neighbor(s) to each node and the maximum capacity gain is attained. If the group size is independent of the size of the network ( $m=\Theta(1)$ ), the order capacity is the same as for unicasting.

Fig. 2(a) is the first region in capacity for both SPR and MPR. When $m=k=\Theta(1)$, the left plot in this figure is very similar to the results derived in [1]. Another interesting observation is the fact that unlike SPR that increasing capacity results in increasing delay, we can increase capacity and decrease delay simultaneously with MPR. This is a significant advantage of using MPR and stems from the fact that MPR embraces interference and consequently, we do not need to sacrifice capacity or delay to improve the other parameter.

Fig. 2(b) shows the capacity-delay tradeoff in the second capacity region for only SPR. For the case of MPR, the capacity or delay is not a function of $R(n)$ and therefore, there is no tradeoff and therefore, we did not plot that result. For this case in SPR, increasing $r(n)$ decreases capacity but has no effect on the delay.

Fig. 2(c) is the third region of capacity for SPR and MPR. This is the broadcasting region of capacity for SPR and MPR and it is clear from this figure that SPR does not provide any tradeoff. In general, by increasing the transmission range we can decrease delay while the capacity remains constant. The reason for this behavior is the fact that all nodes in broadcasting region are receiving the packet and increasing transmission range does not create any interference. On the other hand, when we use MPR and increase the receiver range, again both capacity and delay are improved similar to the first case. Clearly, the capacity of the network with MPR is maximized if we maximize the number of simultaneous transmissions in the network. Ideally, if the receiver range can be made $\Theta(1)$, then a network using MPR can scale linearly with $n$. Obviously, the receiver range is restricted in practice by the complexity of the receivers. However, even with the minimum value for the receiver range, which is the connectivity criterion, MPR still renders a capacity gain compared to SPR. Furthermore, this gain is still an order gain equal to $\Theta(\log n)$ compared to the capacity attained with SPR for $(n, m, k)$-casting.

In summary, the tradeoff between capacity $C_{m, k}(n)$ and delay $D_{m, k}(n)$ with MPR is in sharp contrast to SPR. The results in this paper provide new directions and opportunities for future research activities in wireless ad hoc networks. Another important aspect that we did not discuss in this paper is related to practical limitations and decoding complexity that we can have with MPR scheme. This aspect is important and its investigation is the subject of future studies.

\section{ACKNOWLEDGMENTS}

This work was partially sponsored by the U.S. Army Research Office under grants W911NF-04-1-0224 and W911NF-05-1-0246, by the National Science Foundation under grant CCF-0729230, by the Defense Advanced Research Projects Agency through Air Force Research Laboratory Contract FA8750-07-C-0169, and by the Baskin Chair of Computer Engineering. The views and conclusions contained in this document are those of the authors and should not be interpreted as representing the official policies, either expressed or implied, of the U.S. Government.

\section{REFERENCES}

[1] A. El Gamal, J. Mammen, B. Prabhakar, and D. Shah, "Optimal throughput-delay scaling in wireless networks part i: The fluid model," IEEE Transactions on Information Theory, vol. 52, no. 6, pp. 2568-2592, 2007.

[2] P. Gupta and P. R. Kumar, "The capacity of wireless networks," IEEE Transactions on Information Theory, vol. 46, no. 2, pp. 388-404, 2000.

[3] A. Ozgur, O. Leveque, and D. Tse, "Hierarchical cooperation achieves optimal capacity scaling in ad hoc networks," IEEE Transactions on Information Theory, vol. 53, no. 10, pp. 2549-3572, 2007.

[4] J. J. Garcia-Luna-Aceves, H. R. Sadjadpour, and Z. Wang, "Challenges: Towards truly scalable ad hoc networks," in Proc. of ACM MobiCom 2007, Montreal, Quebec, Canada, September 9-14 2007.

[5] X.-Y. Li, S.-J. Tang, and O. Frieder, "Multicast capacity for large scale wireless ad hoc networks," in Proc. of ACM MobiCom 2007, Montreal, Canada, September 9-14 2007.

[6] A. Keshavarz, V. Ribeiro, and R. Riedi, "Broadcast capacity in multihop wireless networks," in Proc. of ACM MobiCom 2006, Los Angeles, California, USA., September 23-29 2006.

[7] Z. Wang, H. R. Sadjadpour, and J. J. Garcia-Luna-Aceves, "A unifying perspective on the capacity of wireless ad hoc networks," in IEEE INFOCOM 2008, Phoenix, Arizona, USA., April 13-18 2008.

[8] S. Ghez, S. Verdu, and S. Schwartz, "Stability properties of slotted aloha with multipacket reception capability," IEEE Transactions on Automatic Control, vol. 33, no. 7, pp. 640-649, 1988.

[9] S. Kulkarni and P. Viswanath, "A deterministic approach to throughput scaling wireless networks," IEEE Transactions on Information Theory, vol. 50, no. 6, pp. 1041-1049, 2004. 